\title{
Nanoporous Structure in Low-Dielectric Films with Positronium Annihilation Lifetime Spectroscopy
}

\author{
HU Yi-Fan(胡一帆) ${ }^{1 *}$, SUN Jia-Ning ${ }^{2}$, D. W. Gidley ${ }^{2}$ \\ ${ }^{1}$ Department of Physics, Huazhong University of Science and Technology, Wuhan 430074 \\ ${ }^{2}$ Department of Physics, University of Michigan, Ann Arbor, MI 48109, USA
}

\section{(Received 28 February 2005)}

\begin{abstract}
We investigate nano-porous structures in thin low-dielectric films, i.e. the pore sizes, distributions, and interconnectivity, by using depth profiled positronium annihilation lifetime spectroscopy (PALS). It is found that PALS has good sensitivity to probe both interconnected and closed pores in the range from $0.3 \mathrm{~nm}$ to $30 \mathrm{~nm}$, even in the film buried beneath a diffusion barrier. A series of low dielectric constant films of organosilicon-silsequioxane with different weight percentages of porogen have been comparatively investigated. The PALS technique can be used to distinguish the open porosity from the closed one, to determine the pore size, and to detect the percolation threshold with the increasing porosity that represents the transition from closed pores to interconnected pores. Furthermore, the pore percolation length can be derived.
\end{abstract}

PACS: $68.55 . J k, 78.70 . \mathrm{Bj}$

In recent years, resistance-capacitance (RC) delay in the multilevel interconnections of ultra-largescale integrated circuits (ULSIs) has become a dominant factor in overall chip performance. Researchers have taken extensive efforts to develop low-dielectric constant materials to reduce the RC delay. ${ }^{[1-5]}$ Silsequioxane (SSQ) has been intensively investigated as an on-chip low- $k$ material. One reason for this is that it is difficult to obtain good mechanical and thermal stability using pure organic or inorganic materials. The dielectric constant of dense SSQ is usually less than 3 due to the reduced cross-linking density and lower material density when compared to conventional silica $(k \approx 4)$. As the feature size of IC shrink below $0.10 \mu \mathrm{m}$, nano-porosity is being incorporated into SSQ materials to produce ultra low- $k$ films $(k<2.2)$. Understanding the pore structure, such as pore size distribution and pore interconnectivity in porous low$k$ films, is crucial since these parameters are fundamentally important in determining the electrical and mechanical properties. Unfortunately, there are relatively few techniques that are capable of probing pore characteristics in sub-micron films on thick substrates. Recently, beam-based positronium annihilation lifetime spectroscopy (PALS) ${ }^{[6,7]}$ has been demonstrated to be powerful in determining interconnected or closed porous structures in SSQ films. The advantages of the PALS technique are as follows: firstly, PALS is non-destructive and sensitive to pore diameters of $0.3-$ $30 \mathrm{~nm}$; secondly, beam-based PALS is capable of depth profiling multi-layer porous films. In this study, PALS is used to detect the nano-porous structures in porous SSQ low- $k$ films.

By adopting the PALS with thin films, an electrostatically focused beam of several-keV positrons is generated in a high vacuum system using a radioactive beta-decay source of ${ }^{22} \mathrm{Na}$ (Fig. 1). This beam is deflected onto a target sample as shown in Fig. 1(b) and forms positronium ( $\mathrm{Ps}$, the electron-positron bound state). Ps inherently localizes in the pores where its natural annihilation lifetime of $140 \mathrm{~ns}$ is reduced by annihilation with molecular electrons during collisions with the pore surface. The reduced Ps lifetime is correlated with void size and forms the basis the technique.

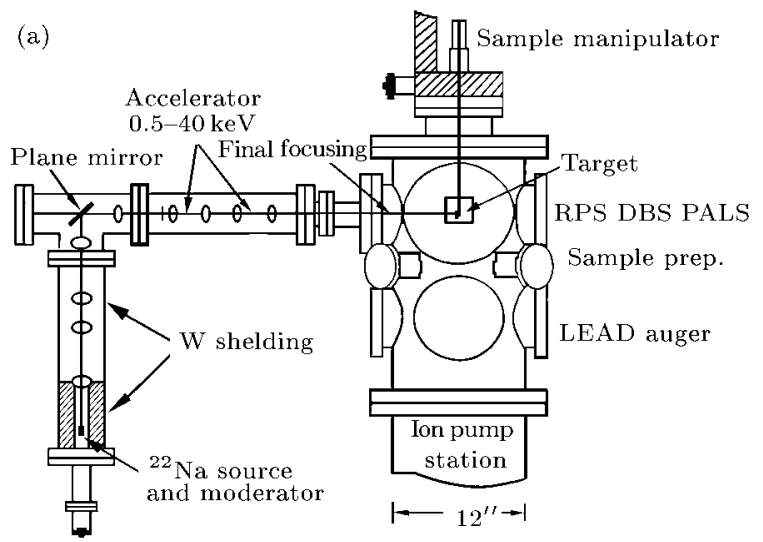

(b)

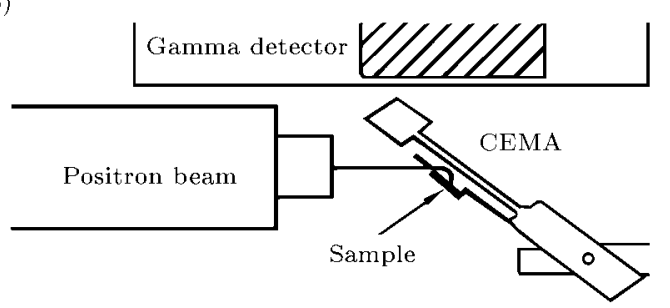

Fig. 1. (a) Schematic diagram of a depth-profiled positron spectrometer at the University of Michigan. (b) Enlarged target chamber and the sample holder. 


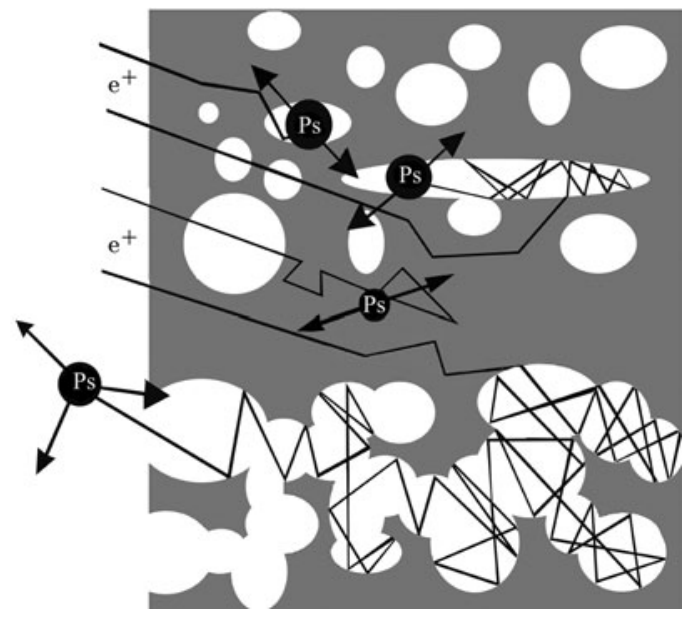

Fig. 2. Positronium formation in the porous material.

The formation of Ps in a porous insulator is described in Fig. 2. After the Ps formation, it begins to diffuse and thermalize in the insulator. When it is thermalized in the pores, Ps is still colliding with the pore walls and the resulting Ps lifetime is shortened by positron annihilation with molecularly bound electrons in addition to the captured electron. Furthermore, Ps may diffuse over a long distance that can be greater than the porous film thickness if the pores are interconnected. As a result, Ps can easily diffuse out of the film and into the surrounding vacuum as depicted as Fig. 2.

A series of blanket nanoporous low-dielectric films on silicon wafers were prepared. The thin films were processed by spin coating a sol onto a silicon wafer, treating the as-spun film with moist ammonia, and thermally curing (hardening) the film. The initial sol was comprised of silsesquioxane resin and two solvents: methyl propyl ketone and tetradecane. Methyl propyl ketone is a low boiling-point solvent, which was used to control the thickness of the as-spun film. The second solvent, tetradecane, which has a high boiling point, remained with the resin in the as-spun film. The ratio of the resin to high-boiling point solvent tetradecane is adjusted to control the porosity and hence to control the dielectric constant of the film (and the average pore size). After spin coating, the as-spun film was exposed to moist ammonia to cause the film to gel in the presence of the tetradecane solvent. A hot-plate step of $150^{\circ} \mathrm{C}$ is used to evaporate the lowboiling-point methyl propyl ketone solvent. The remaining tetradecane was then removed by subsequent heating steps, which included the final cure in the range $400-500^{\circ} \mathrm{C}$. These films are about $1 \mu \mathrm{m}$ thick, with dielectric constant $k$ varying from 1.7 to 2.5 .

These porous SSQ (silsesquioxane) films with different weight percentages of porogen (ranges from 0 $50 \%$ ) are investigated using positronium annihilation lifetime spectroscopy. The sample preparation pro- cedure has been described elsewhere. ${ }^{[6]}$ Beam-PALS testing was performed at room temperature to probe the structure of the non-porous and the porous SSQ films at a positron beam implantation energy of 1.0, 3.0, 5.0, $8.0 \mathrm{keV}$. The intensities of the Ps annihilation in the film, $I_{\mathrm{o}-\mathrm{Ps}}$, include Ps in both meso-pores and micro-pores. Ps can exist in two different spin states: ortho- and para-states. Ortho-positronium (O-Ps) is more stable and localizes in the void volume. The meso-pore diameter ranges from $2-50 \mathrm{~nm}$, which is determined by the O-Ps lifetime. The corresponding O-Ps lifetime for the meso-pore is $30-41 \mathrm{~ns} .{ }^{[10]}$ The micro-pore diameter is less than $2 \mathrm{~nm}$ and the corresponding O-Ps lifetime is shorter than $30 \mathrm{~ns}$. The vacuum intensity consists of two parts: the first part simply comes from backscattered Ps $(\sim 2 \%)$ in the case of close pores, and the second part comes from escaped Ps in the case of open pores. For the total vacuum intensity, the corresponding O-Ps lifetime is about $140 \mathrm{~ns}$. Therefore, we can distinguish the pore types in the films by the vacuum intensity (with $140 \mathrm{~ns}$ lifetime) and the Ps intensity (with $<40$ ns lifetime) in the film. When the vacuum intensity is much higher than $2 \%$, and the in-film Ps intensity is low, the pores are considered as open pores. Conversely, when the vacuum intensity is $2 \%$ or just slightly higher than $2 \%$ (still very low), and the in-film Ps intensity is high, the pores are considered as closed pores. Usually, the pores in low- $k$ film are partially opened, which means that some pores near the surface are connected with the vacuum and other pores berried in the deep film are closed. At low positron implantation energies, the Ps intensity in vacuum is higher than $2 \%$, which indicates that the Ps intensity in vacuum includes not only the backscattered Ps but also some Ps escaped from some open pores near the surface. As the positron implantation energy increases, the Ps intensity in vacuum decreases, and finally it reaches $2 \%$, which indicates that the implanted positrons move far away so that they cannot probe the near-surface pores.

The in-film intensities and in-vacuum intensities of Ps are illustrated in Fig. 3. From this paragraph, we can compare the trends of the in-film intensities and in-vacuum intensities at different positron implantation energies. As can be seen, when the porogen loading (porogen weight fraction) is zero, the in-film intensity is almost zero and the in-vacuum intensity results solely from surface backscattered Ps which is dependent on the positron implantation energies, since no micro-pore or meso-pore can be formed. For example, when the positron implantation energy is $1 \mathrm{keV}$ and the vacuum intensity is $8 \%$, we can still consider that most pores are closed because the in-vacuum intensity keeps constant as the porogen loading increases $(<55 \%)$. As the positron implantation energy increases, the vacuum intensity decreases rapidly. At 
low porogen loading the in-film intensity (the corresponding lifetime is $30-41 \mathrm{~ns}$ ) results from Ps annihilating in closed pores and the vacuum Ps intensity is consistent with backscattered Ps and escaped Ps near the surfaces for the samples with porogen loading below $50 \mathrm{wt} . \%$, indicating that there are mainly closed (not all) pores in these lower porosity films. The amount of Ps that annihilates in the film keeps nominally constant. A sudden increase in the Ps annihilation fraction in vacuum occurs when the porogen is increased to $55 \mathrm{wt} . \%$ and this is accompanied by a sharp decrease in the in-film Ps intensity. Clearly, some Ps formed in the film has diffused into vacuum as the porogen loading reaches above $55 \mathrm{wt} . \%$. Therefore, $50 \mathrm{wt} . \%$ would be a lower limit value of the pore percolation threshold. At the threshold, the nanopores start to merge or connect with the surrounding pores and, as a result, open paths to the free surface can be formed. Some Ps is then able to diffuse into vacuum through the open channels and to produce a higher than normal (backscattered Ps only) signal in the vacuum. As the porogen weight fraction is increased to $60 \mathrm{wt} . \%$, the porous network is totally interconnected, as supported by the fact that all the Ps intensity in
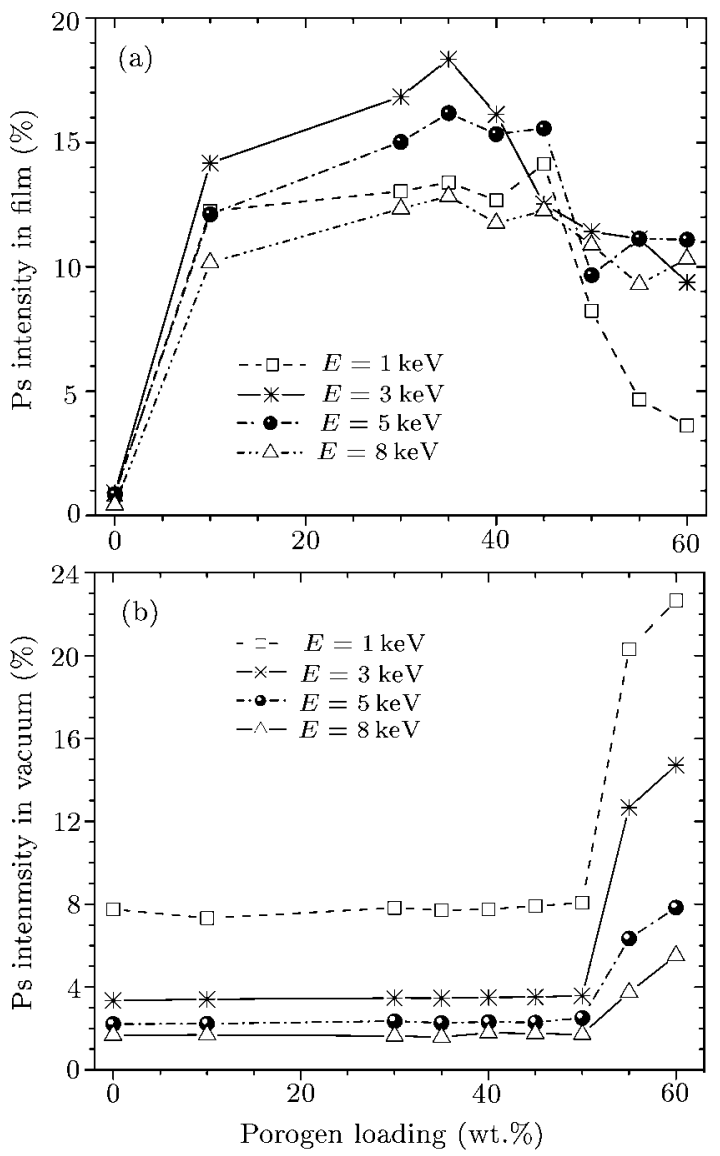

Fig. 3. Ps intensities in the porous SSQ films (a) and in vacuum (b) versus porogen weight fraction (porogen loading) with different positron implantation energy. vacuum increases sharply, which means that all Ps in mesopores escapes into vacuum. Below the percolation threshold, all the pores generated in these films are closed and isolated.

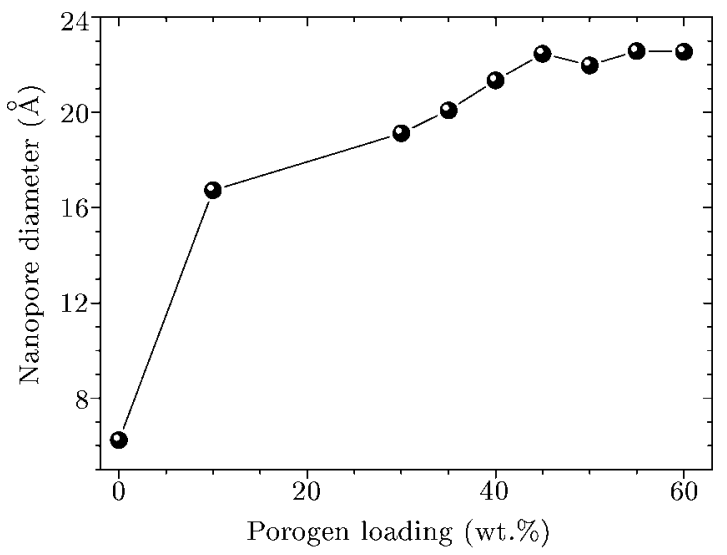

Fig. 4. Pore size versus porogen loading, calculated from the result of Ref. [10].

A pore size distribution can be derived as described in Ref. [10] and will not be discussed here. In order to investigate the microstructure of the "nanopores", it is important to calibrate Ps lifetimes with pore sizes. In a very large pore (non-quantum mechanical) regime (pores with mean free paths in order of $100 \mathrm{~nm}$ ), calibration is usually performed using very high porosity (90-98\%) silica powders. In the sub-nm pores, the quantum mechanical model first developed by Tao ${ }^{[8]}$ and Eldrup et al. ${ }^{[9]}$ has been empirically used to calibrate Ps lifetimes of several nanoseconds in pore size, such as those in polymers. In a simple model,,${ }^{[, 9]}$ Ps was localized in a spherical infinitely deep potential well and was only annihilated with molecular electrons when its position was a short distance from the pore surface. With only the ground state of Ps considered in the Tao-Eldrup model, it is insufficient to characterize larger voids where the pore diameter approaches the thermal De Broglie wavelength of positronium (about $6 \mathrm{~nm}$ ). Thermally excited states of Ps atoms in the pores must be included in the calculation. As a result, the effect of the sample temperature should also appear in the calibration of lifetime versus pore size. To extend the quantum model fully to the classical large-pore limit, Dull et al. ${ }^{[10]}$ have modified the Tao-Eldrup model to characterize both micro and meso-pores. In Ref. [10], a rectangular pore shape was adopted for calculation simplicity and the authors assumed that there is no Ps-surface interaction and the Ps atoms can randomly sample all of the states in the rectangular well with a probability governed by the Maxwell-Boltzmann distribution. At a given temperature, four lifetime curves versus pore dimensions were calculated for different temperatures. ${ }^{[10]}$ Based on the room-temperature curve in Ref. [10], we calcu- 
late the average sizes of the nano-pores versus porogen loading, as shown Fig. 4. It is obvious that the pores become larger in size as the porogen loading increases. In the present porous SSQ films, some Ps (but not all) can escape into vacuum for the films just above the percolation threshold. This indicates that the Ps diffusion length in the porous network is limited near the threshold by pore morphology. Since Ps can diffuse over great distances in fully connected pore networks, we assume that short Ps diffusion lengths near the threshold can denote the scale length over which pores have percolated. We call it the percolation length and make it equal to the deduced value of the Ps diffusion length. The Ps diffusion length for a given film is determined by depth profiling. As an example, we use a porous SSQ film supplied by Sematech, which clearly indicates the frustration of Ps diffusion just above the percolation threshold of this material. In Sematech's samples, the pores are also partially opened. At low positron implantation energies, the Ps intensity in vacuum (with 140 ns lifetime) is also higher than $2 \%$. However, as the positron implantation energy increase to a certain point, the Ps intensity in vacuum quickly returns back to $2 \%$. Since each positron-implantation energy corresponds to an average implantation distance from the surface, we can conclude that a threshold of the positron implantation energy exists. When the positron implantation energy is higher than the threshold, the implantation depth is longer than the positronium diffusion length, and thus Ps cannot escape into vacuum. Therefore, the corresponding implantation-depth energy threshold can be defined as the positronium diffusion length or the percolation length. The depth-profiled results of Ps intensity, as shown in Fig. 5, show that less Ps is able to diffuse into vacuum at higher positron implantation energies (deeper implantation), while more annihilates in the film. This suggests that pores in a deep film are not connected to vacuum.

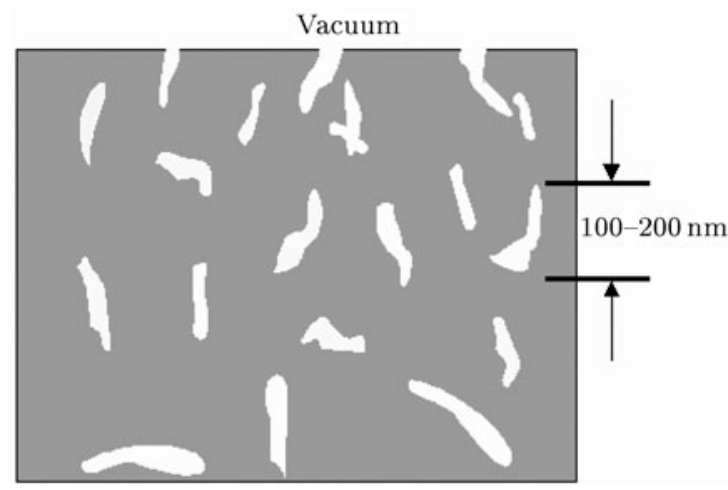

Fig. 5. Percolation length determined to be $100-200 \mathrm{~nm}$.
Using standard models ${ }^{[11]}$ for Ps implantation and diffusion, we determine a diffusion length of about $150 \mathrm{~nm}$ in this particular film with a length over which the pores are interconnected. For comparison, the infilm Ps lifetime around $40 \mathrm{~ns}$ corresponds to a cylindrical pore diameter (and mean free path) of $2.3 \mathrm{~nm}$. Thus we can visualize long but finite tubular pores that are closed on the 100-200-nm length scale.

In summary, PALS is a non-destructive depthprofiling technique with rather simple requirement that positrons can be implanted into the porous film where Ps can form. It is sensitive to all the void volume greater than a few angstroms in size, regardless of whether the pores are open or closed. The technique can be used to distinguish the open porosity from the closed porosity and can be utilized as a simple technique to determine porous structures. Also, PALS can be used to determine the percolation threshold and to deduce the pore percolation length. In open and partially interconnected pores, Ps can easily escape into vacuum. This feature has been exploited to determine the pore percolation threshold. A consistent percolation threshold has been determined by using various beam implantation energies. Above the percolation threshold, the porous network structure and the percolation length, which is defined as the Ps diffusion length in the porous network, can frustrate Ps diffusion into vacuum. The Ps diffusion length can be determined by depth profiling of each film.

\section{References}

[1] 1999 International Technology Roadmaps of Semiconductors (San Jose, CA: American Semiconductor Industry Association)

[2] Hedrick J L, Cha H J, Miller R D, Yoon D Y, Brown H R, Srinivasan S, Pietro R D, Cook R F, Hummel J P, Klaus D $\mathrm{P}$, Liniger E G and Simonyi E E 1997 Macromolecules 30 8512

[3] Fan H, Bentley H R, Kathan K R, Clem P, Lu Y and Brinker C J 2001 J. Non-Cryst. Solids. 28579

[4] Maex K, Baklanor M R, Shamiryan D, Lacopi F, Brongersma S H and Yanovitskaya Z S 2003 J. Appl. Phys. 938793

[5] Pazzeri P, Rubloff G W, Vanzetti L, Briber R M, Anderle M, Bersani M, Park J J, Kim H C, Volksen W, Miller R D and Lin Z 2004 Surf. Interf. Anal. 36304

[6] Yang S, Mirau P A, Pai C S, Nalamasu O, Reichmanis E, Pai J C, Obeng Y S, Seputro J, Lin E K, Lee H J, Sun J N and Gidley D G 2002 Chem. Mater. 14369

[7] Gidley D W, Frieze W E, Yee A F, Dull T L, Ho H M and Ryan E T 1999 Phys. Rev. B 60 R5157

[8] Tao S J 1972 J. Chem. Phys. 565499

[9] Eldrup M, Lighbody D and Sherwood J N 1981 Chem. Phys. 6351

[10] Dull T L, Frieze W E, Gidley D W, Sun J N and Yee A F 2001 J. Phys. Chem. B 1054657

[11] Schultz P J and Lynn K G 1988 Rev. Mod. Phys. 60701 